\title{
Inventário estruturado de formigas (Hymenoptera, Formicidae) em floresta ombrófila de encosta na ilha da Marambaia, $\mathbf{R J}$
}

\author{
Michel de S. Schütte ${ }^{1}$, Jarbas M. Queiroz ${ }^{1}$, Antônio J. Mayhé-Nunes ${ }^{2}$ \& Marcos Paulo dos S. Pereira ${ }^{1}$ \\ 1. Departamento de Ciências Ambientais, Instituto de Florestas, Universidade Federal Rural do Rio de Janeiro, Caixa Postal 74.514 , \\ 23890-971 Seropédica, RJ, Brasil. (jarbas@ufrrj.br) \\ 2. Departamento de Biologia Animal, Instituto de Biologia, UFRRJ, Rod. BR 465, Km 7,23890-000 Seropédica, RJ, Brasil. (amayhe@ufrrj.br)
}

\begin{abstract}
Structured inventory of ants (Hymenoptera, Formicidae) in atlantic slope rain-forest of Marambaia Island, RJ. Ants are an important functional component in tropical forest due to their ecological roles, biomass and species diversity. Although the Atlantic Forest is one of the best studied ecosystems in Brazil, there is a lack of information about ant diversity in forest fragments of the state of Rio de Janeiro. The composition and richness of the ant fauna from atlantic slope rain-forest in Marambaia island-RJ were assessed by the structured inventory in an area of 0.6 ha. Pitfalls traps and hand collecting were used for sampling ants in the litter and on vegetation from January to July/2004. A total of 29 genera and 82 species were found in this survey. Ant abundance and richness were higher in March than in July samples. Eveness and diversity of ants in the samples were not affected by the time of the year. Collections of ants from dead twigs yielded an additional seven species and provided information about the ant biology. Sampling ants on plants resulted in 32 species but 12 species were not recorded in litter samples. Some species were recorded exclusively on vegetation, such as Pseudomyrmex, Crematogaster and Pachychondyla species. This study aims to contribute to the development of conservation priorities in one of the most threatened ecosystems in the world.
\end{abstract}

KEYWORDS. Island fauna, canopy ants, litter, biodiversity, Brazil.

\begin{abstract}
RESUMO. As formigas são componentes funcionais importantes em florestas tropicais devido aos papéis ecológicos que exercem, à grande biomassa e à riqueza de espécies. Embora a Mata Atlântica seja um dos ecossistemas mais bem estudados no Brasil, ainda faltam informações sobre a diversidade de formigas nos fragmentos florestais do Estado do Rio de Janeiro. A riqueza e composição da assembléia de formigas em floresta ombrófila de encosta na ilha da Marambaia (RJ) foi estudada através de um inventário estruturado em uma área de 0,6 ha. Armadilhas do tipo "pitfall" e coletas manuais foram empregadas na serapilheira e sobre a vegetação entre os meses de janeiro e julho de 2004. Um total de 29 gêneros e 82 espécies foi encontrado na amostragem. A abundância e a riqueza de espécies foram maiores nas amostras de março do que de julho. Já a eqüitatividade e diversidade de formigas nas amostras não foram influenciadas pela época da coleta. As amostras de formigas em galhos mortos adicionaram seis espécies à lista, acrescentando informações sobre a biologia das espécies. As amostras sobre plantas totalizaram 32 espécies de formigas, das quais 12 foram exclusivas, como as espécies de Pseudomyrmex e algumas de Crematogaster e Pachycondyla. Este estudo pretende contribuir para o desenvolvimento de prioridades conservacionistas em um dos ecossistemas mais ameaçados do mundo.
\end{abstract}

PALAVRAS-CHAVE. Fauna insular, formigas de dossel, serapilheira, biodiversidade, Brasil.

Dentre a extraordinária diversidade de insetos nas regiões tropicais, as formigas compõem um grupo de destaque. Esses organismos apresentam grande riqueza em espécies e funções ecológicas nos ecossistemas (HÖLLDOBLER \& WILSON, 1990). Muitas são detritívoras, outras são predadoras ou se alimentam de produtos vegetais como néctar e sementes e há ainda um grupo especializado, os cultivadores de fungo da tribo Attini, a qual inclui algumas espécies que podem causar severas desfolhas às plantas (Schultz \& McGlynn, 2000). Sua sensibilidade às mudanças no ambiente e sua relação com a estrutura das comunidades de outros organismos fazem das formigas potenciais indicadores ambientais (MAJER, 1997).

Estudos concentrados em formigas que vivem na serapilheira demonstram que a utilização de mais de uma técnica de amostragem é mais eficiente que a utilização de uma única técnica para realizar inventários completos das espécies (MAJer, 1997; Delabie et al., 2000b). Além disso, inventários estruturados que utilizam diferentes métodos de coleta e coletas em diferentes ambientes ou épocas do ano são mais apropriados para estudos ecológicos, pois os dados quantitativos podem ser aplicados para a caracterização da comunidade sob diferentes condições (Longino \& Colwell, 1997). Em comparação ao grande número de trabalhos enfocando a comunidade de serapilheira, poucos estudos tentaram amostrar a comunidade de formigas sobre plantas. Um dos problemas para esse grupo é justamente a amostragem da fauna arborícola, cuja principal técnica continua sendo a nebulização com inseticidas piretróides (ADIs et al., 1984). Amostras da fauna arborícola são importantes porque, em todas as regiões tropicais e subtropicais do mundo, as formigas aparecem como o maior componente deste habitat (DAvIDSON et al., 2003), havendo grande potencial para a descoberta de espécies novas (LEWINSOHN et al., 2005).

A conservação da biodiversidade da Mata Atlântica tem especial relevância por ser este um bioma prioritário em termos de conservação em nível global, com elevada diversidade e endemismo (MYers et al., 2000). O Estado do Rio de Janeiro destaca-se por ter vários remanescentes de Mata Atlântica, abrigando grande diversidade para diferentes grupos da flora e fauna e é um dos maiores centros de endemismo do país. Essas características, aliadas à pressão de grandes centros 
urbanos próximos aos remanescentes florestais do estado, fazem com que este possa ser considerado um hotspot dentro do grande hotspot Mata Atlântica (RocHA et al., 2003). A ilha da Marambaia, Rio de Janeiro, que faz parte do bloco de remanescentes florestais da Região Sul-Fluminense, é considerada como área prioritária para a conservação (MMA/SBF, 2003).

Embora estudos sobre assembléias de formigas já tenham sido conduzidos em outros locais de Mata Atlântica (DELABIE et al., 2000b), faltam informações sobre a diversidade deste grupo nos remanescentes florestais do Estado do Rio de Janeiro. MEneguete et al. (1999) realizaram um estudo na ilha da Marambaia com objetivo de testar diferentes tipos de iscas para atração de formigas. Os autores coletaram as formigas na praia da Armação, em área de restinga. No presente trabalho, o objetivo foi realizar um inventário estruturado de formigas em floresta ombrófila de encosta nesta ilha utilizando duas técnicas básicas de coleta para amostrar os diferentes microhabitats e épocas do ano.

\section{MATERIAL E MÉTODOS}

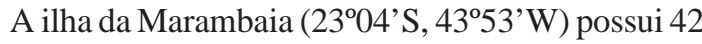
$\mathrm{km}^{2}$ de área, apresentando relevos que variam entre baixada, meia baixada e elevação rochosa (pico em $641 \mathrm{~m}$ ) e com áreas de restinga e floresta (PEREIRA et al., 1990). A ilha está ligada ao continente por uma estreita faixa de terra, que juntas delimitam a baía de Sepetiba. O local das coletas é uma floresta ombrófila submontana, caracterizando-se por apresentar um dossel de aproximadamente $25 \mathrm{~m}$ de altura, com alguns indivíduos alcançando cerca de $35 \mathrm{~m}$. As espécies arbóreas mais comuns são Guapira opposita (Vell.) Reitz (Nyctaginaceae), Nectandra oppositifolia Nees (Lauraceae), Vochysia oppugnata (Vell.) Warm (Vochysiaceae) e Pseudopiptadenia contorta (DC.) M. P. Lima (Fabaceae). O sub-bosque possui árvores de 8 a $12 \mathrm{~m}$ e lianas lenhosas que se ramificam, expondo suas folhas somente no dossel superior. É comum na região a ocorrência de chuvas orográficas, havendo excedente hídrico em todos os meses do ano. Entre novembro e março ocorrem as maiores precipitações (acima de 200 mm mensais) e os meses mais secos vão de junho a setembro (entre 50 e 100 mm mensais). Já as temperaturas médias mensais variam ao longo do ano com amplitude máxima de $6{ }^{\circ} \mathrm{C}$, sendo a média do mês mais frio de $19^{\circ} \mathrm{C}$ (julho) e do mês mais quente de $25^{\circ} \mathrm{C}$ (fevereiro) (PEREIRA et al., 1990).

Para o estudo da fauna de formigas, foram utilizadas duas técnicas básicas de coleta: armadilhas do tipo pitfall no solo e na copa (MAJER, 1997) e coleta manual, com ou sem o uso de iscas. As coletas foram realizadas entre janeiro e julho de 2004. Três parcelas de $2000 \mathrm{~m}^{2}$ cada foram delimitadas no interior da mata, totalizando 6000 $\mathrm{m}^{2}$ de área amostral. As parcelas estavam em altitudes de $217 \mathrm{~m}, 322 \mathrm{~m}$ e $440 \mathrm{~m}$, distantes entre si aproximadamente $200 \mathrm{~m}$ ao longo de uma encosta localizada no lado oeste da ilha voltada para a baía de Sepetiba. As armadilhas foram instaladas no mês de janeiro, permanecendo na área até a data da última coleta, quando foram trazidas de volta ao laboratório.
Serapilheira. Foram realizadas três coletas com armadilhas no solo (12 de janeiro, 30 de março e 25 de julho). Para cada coleta, foram utilizadas 60 armadilhas, feitas de garrafas PET recicladas, com isca de sardinha conservada em óleo, para atração. A sardinha foi posta internamente ao funil que cobriu a parte superior da armadilha. Essas armadilhas tinham 9,5 cm de diâmetro por $16 \mathrm{~cm}$ de altura e foram preenchidas com $100 \mathrm{ml}$ de formalina a $3 \%$ para conservar o material coletado. Dentro de cada uma das parcelas, seguindo um protocolo padrão de coletas (Agosti \& Alonso, 2000), 20 armadilhas foram instaladas, eqüidistantes $10 \mathrm{~m}$ entre si, permanecendo ativadas por 48 horas.

A coleta manual de formigas na serapilheira, com o uso de isca de sardinha, foi realizada em 01 de maio. Uma pequena porção de sardinha, $1 \mathrm{~cm}^{3}$, foi disposta no centro de quadrados de papel branco de $120 \mathrm{~cm}^{2}$, eqüidistantes $10 \mathrm{~m}$ entre si, sendo 20 em cada parcela, com repetição de uma das parcelas durante a noite. As iscas permaneceram expostas por cerca de 1 hora, sendo realizadas duas coletas com intervalo de meia hora. Coletou-se, com pinça entomológica, todas as espécies sobre e sob a área de papel.

Para a amostragem de formigas que nidificam em galhos, foi feita uma coleta em galhos grossos $(5,1$ a 15 $\mathrm{cm}$ de diâmetro) (02 de maio) e uma em galhos finos ( 0,3 a $5 \mathrm{~cm}$ de diâmetro) (25 de julho). Para a coleta de galhos grossos foram distribuídas, em cada parcela, quatro subparcelas $(20 \times 2 \mathrm{~m})$ paralelas, eqüidistantes $10 \mathrm{~m}$ entre si, totalizando 12 subparcelas na área amostral. Dentro de cada subparcela, os galhos encontrados foram processados no campo, quando foram coletadas manualmente as colônias de formigas que utilizavam este sítio para nidificação bem como outras eventuais forrageiras no interior dos galhos. Para todas as colônias foi registrada a presença de sexuados, operárias e imaturos. Para coleta de galhos finos foram selecionadas 10 subparcelas de $1 \mathrm{~m}^{2}$, eqüidistantes $10 \mathrm{~m}$ entre si, totalizando 30 subparcelas na área amostral. No campo, todos os galhos de cada subparcela foram coletados manualmente e acondicionados em sacos plásticos, fechados e etiquetados com referência do ponto de coleta. No laboratório, as amostras foram processadas para a coleta manual das colônias de formigas que utilizavam este sítio para nidificação e outras eventuais forrageiras no interior dos galhos.

Sub-bosque. Foi feita uma coleta manual de formigas sobre as plantas do sub-bosque (15 de julho). Utilizou-se um transecto de $50 \mathrm{~m}$ dentro de cada parcela, amostrando-se todas as plantas entre 1 e 1,5 m de altura, até 1 metro de distância da linha central para cada um dos lados. As plantas foram inspecionadas durante cinco minutos, coletando-se manualmente as espécies de formigas com auxílio de pinça entomológica.

Copa. Para acessar as espécies de formigas na copa das árvores foram realizadas duas coletas com o uso de pitfalls içados (12 de janeiro e 30 de março). Nessas coletas as armadilhas eram semelhantes às utilizadas no solo. Elas foram instaladas sem a necessidade de se escalar a árvore, levantando-as até a copa, com o auxílio de uma linha de nylon, até que estivessem em contato com um galho. O critério para a escolha das árvores foi a 
proximidade com os pitfalls no solo, totalizando 60 armadilhas em cada coleta, 20 em cada parcela. As armadilhas permaneceram ativadas por um período de 48 horas.

Foram realizadas duas coletas manuais de formigas sobre o tronco de árvores vivas (11 de janeiro e 29 de março). Para cada uma das coletas foram selecionadas 60 árvores - as mesmas onde ocorrera a coleta de copa - que foram vistoriadas durante cinco minutos. Em cada árvore as formigas foram coletadas com auxílio de pinça entomológica.

Após as coletas, as formigas foram transferidas para recipientes etiquetados com data, tipo de amostragem e ponto de coleta. Após a triagem em laboratório as formigas foram fixadas em pequenos recipientes de vidro, contendo álcool $70 \%$. Posteriormente, estas foram separadas em morfoespécies e montadas em via seca. A identificação das espécies foi feita com o auxílio de chaves dicotômicas e através de comparação com material de coleções. O material coletado foi depositado na Coleção Entomológica Costa Lima (CECL), IB, UFRRJ.

Análise dos dados. Calculou-se a freqüência de ocorrência individualmente para as espécies em cada tipo de amostragem. As amostras de março e julho foram comparadas estatisticamente segundo a riqueza, abundância, diversidade ( $H^{\prime}$ ) e eqüitatividade (Pielou) (MAgurRan, 1988). As armadilhas foram agrupadas por parcela e a comparação foi feita com o teste de Mann-Whitney (ZAR, 1999), considerando três repetições. Nestas análises, a coleta realizada em janeiro foi descartada devido aos possíveis efeitos do impacto causado pelo trabalho de delimitação das parcelas e instalação das armadilhas nesta época (GREensLade, 1973).

\section{RESULTADOS}

Um total de 29 gêneros e 82 morfoespécies foi coletado na ilha da Marambaia, somando 8.532 indivíduos, dividido em oito subfamílias: Myrmicinae (44 espécies), Formicinae (14), Ponerinae (12), Ecitoninae (três), Ectatomminae (três), Pseudomyrmecinae (três), Dolichoderinae (duas), Heteroponerinae (uma) (Tab. I).

Dos parâmetros ecológicos analisados para as formigas capturadas nas armadilhas de solo, a abundância e a riqueza de espécies foram significativamente diferentes entre as amostras de março e julho, já a eqüitatividade (Pielou) e diversidade (H') foram estatisticamente similares (Figs. 1-4).
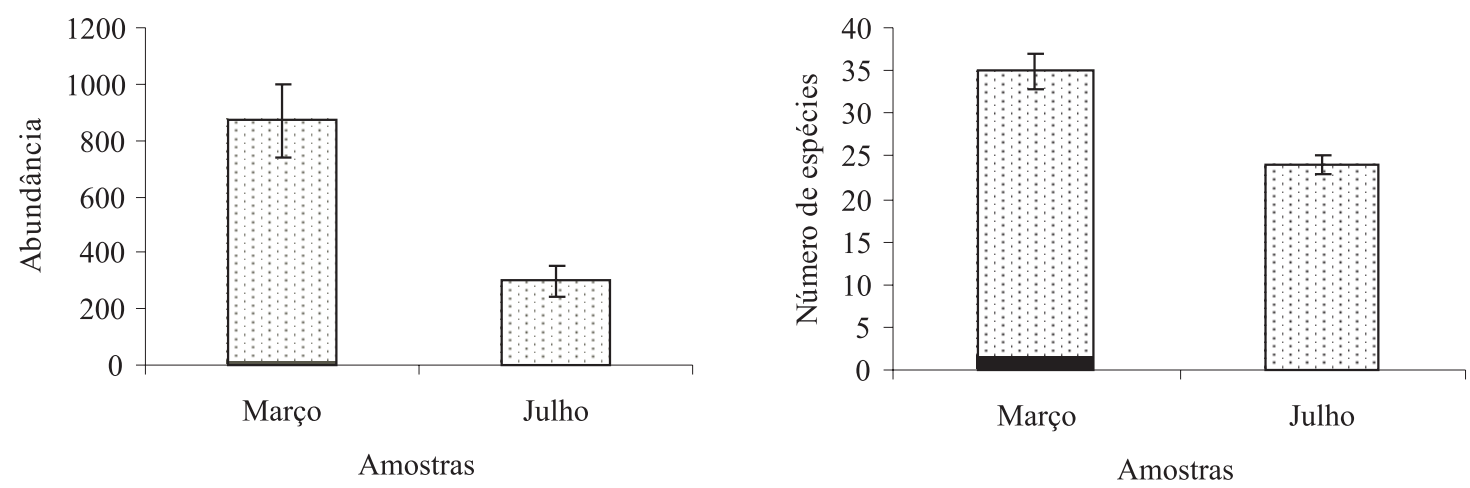

3

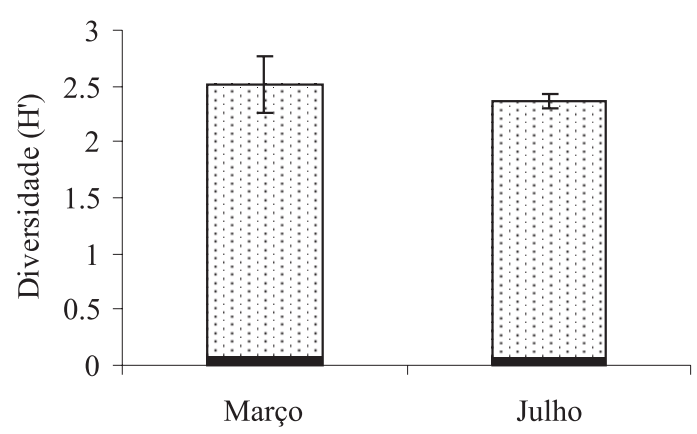

Amostras

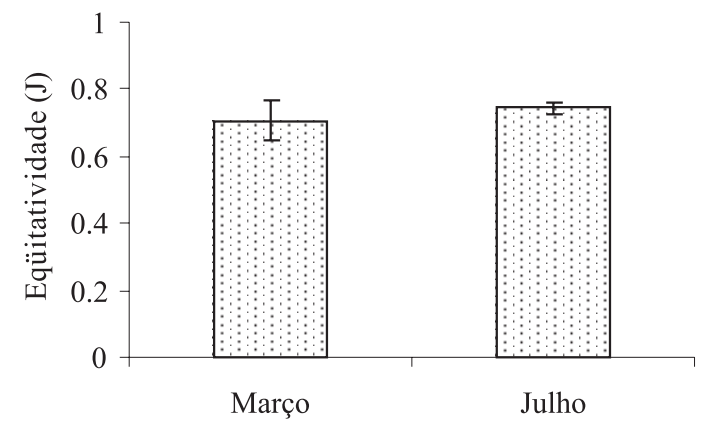

Amostras

Figs. 1-4. Parâmetros ecológicos em amostras de formigas com armadilhas de solo, em duas épocas distintas no ano de 2004, em floresta ombrófila de encosta, na ilha da Marambaia, RJ. 1, abundância de formigas, $\mathrm{P}=0,05 ; 2$, riqueza de espécies, $\mathrm{P}=0,04 ; 3$, índice de diversidade de Shannon, $\mathrm{P}=0,51 ; 4$, eqüitatividade (Pielou), $\mathrm{P}=0,83$. 
Tabela I. Lista das espécies encontradas na mata de encosta da ilha da Marambaia, RJ, com a freqüência de ocorrência (\%) por técnica/ ambiente de amostragem, janeiro a julho/2004 (GF, formigas coletadas em galhos finos; GG, formigas coletadas em galhos grossos; MS, coleta manual no solo com isca; MT, coleta manual no tronco; PC, armadilha na copa; PS, armadilha no solo; SB, formigas coletadas manualmente no sub-bosque).

\begin{tabular}{|c|c|c|c|c|c|c|c|}
\hline Espécies & MS & GF & GG & PS & SB & $\mathrm{PC}$ & M T \\
\hline \multicolumn{8}{|l|}{ MYRMICINAE } \\
\hline Acromyrmex aspersus F. Smith, 1858 & 0 & 0 & 0 & 0 & 0 & 0 & 2,7 \\
\hline Acromyrmex subterraneus brunneus Forel, 1911 & 0 & 0 & 0 & 0,6 & 0 & 0 & 2,7 \\
\hline Apterostigma sp. & 0 & 0 & 0 & 3,9 & 0 & 0 & 0 \\
\hline Carebara sp. & 2,7 & 0 & 0 & 8,9 & 0 & 0 & 0 \\
\hline Cephalotes pallens (Klug, 1824) & 0 & 0 & 0 & 0,6 & 0 & 0 & 0 \\
\hline Crematogaster curvispinosa Mayr, 1862 & 0 & 0 & 0 & 0 & 1,1 & 9,5 & 0 \\
\hline Crematogaster limata F. Smith, 1858 & 0 & 2,7 & 4,8 & 1,7 & 2,2 & 0 & 16,2 \\
\hline Crematogaster nigropilosa Mayr, 1870 & 0 & 0 & 0 & 0 & 16,7 & 0 & 0 \\
\hline Crematogaster tenuicula Forel, 1904 & 0 & 8,1 & 0 & 0 & 2,2 & 0 & 0 \\
\hline Cyphomyrmex sp. & 0 & 0 & 0 & 4,4 & 0 & 0 & 0 \\
\hline Hylomyrma sp. & 0 & 0 & 0 & 1,7 & 0 & 0 & 0 \\
\hline Mycocepurus smithi Forel, 1893 & 0 & 0 & 0 & 5,0 & 0 & 0 & 0 \\
\hline Pheidole sp. 3 & 50,7 & 2,7 & 4,8 & 48,3 & 3,3 & 4,8 & 10,8 \\
\hline Pheidole sp. 4 & 13,3 & 8,1 & 0 & 9,4 & 0 & 0 & 0 \\
\hline Pheidole sp. 5 & 2,7 & 0 & 0 & 13,3 & 0 & 0 & 0 \\
\hline Pheidole sp. 6 & 16,0 & 8,1 & 4,8 & 48,3 & 0 & 0 & 5,4 \\
\hline Pheidole sp. 7 & 1,3 & 8,1 & 0 & 5,6 & 0 & 0 & 0 \\
\hline Pheidole sp. 8 & 10,7 & 0 & 14,3 & 35,0 & 0 & 0 & 0 \\
\hline Pheidole sp. 9 & 0 & 0 & 0 & 1,1 & 0 & 0 & 0 \\
\hline Pheidole sp. 10 & 4,0 & 8,1 & 0 & 10,6 & 1,1 & 0 & 0 \\
\hline Pheidole sp. 11 & 0 & 2,7 & 19,1 & 3,3 & 0 & 4,8 & 5,4 \\
\hline Pheidole sp. 12 & 2,7 & 0 & 0 & 6,7 & 0 & 0 & 0 \\
\hline Pheidole sp. 13 & 8,0 & 16,2 & 9,5 & 23,9 & 0 & 0 & 0 \\
\hline Pheidole sp. 14 & 1,3 & 0 & 0 & 0,6 & 0 & 0 & 0 \\
\hline Pheidole sp. 15 & 0 & 0 & 4,8 & 1,1 & 0 & 0 & 0 \\
\hline Procryptocerus aff. convergens & 0 & 0 & 0 & 0 & 4,4 & 0 & 2,7 \\
\hline Sericomyrmex sp. 1 & 0 & 0 & 0 & 8,3 & 0 & 0 & 0 \\
\hline Sericomyrmex sp. 2 & 0 & 0 & 0 & 3,3 & 0 & 0 & 0 \\
\hline Solenopsis sp. 1 & 0 & 0 & 0 & 16,1 & 0 & 0 & 0 \\
\hline Solenopsis sp. 2 & 0 & 0 & 0 & 2,2 & 0 & 0 & 0 \\
\hline Solenopsis sp. 3 & 4,0 & 0 & 0 & 21,7 & 0 & 0 & 2,7 \\
\hline Solenopsis sp. 4 & 9,3 & 0 & 4,8 & 36,7 & 0 & 4,8 & 0 \\
\hline Solenopsis sp. 5 & 0 & 0 & 0 & 14,4 & 0 & 0 & 0 \\
\hline Solenopsis sp. 6 & 1,3 & 0 & 0 & 11,7 & 0 & 0 & 0 \\
\hline Solenopsis sp. 7 & 1,3 & 8,1 & 9,5 & 11,7 & 0 & 0 & 0 \\
\hline Solenopsis sp. 8 & 2,7 & 0 & 0 & 0,6 & 0 & 0 & 0 \\
\hline Solenopsis sp. 9 & 0 & 0 & 0 & 1,7 & 0 & 0 & 0 \\
\hline Strumigenys sp. 1 & 0 & 0 & 0 & 0,6 & 0 & 0 & 0 \\
\hline Strumigenys sp. 2 & 0 & 0 & 0 & 0,6 & 0 & 0 & 2,7 \\
\hline Trachymyrmex sp. 1 & 0 & 0 & 0 & 2,2 & 0 & 0 & 0 \\
\hline Trachymyrmex sp. 2 & 0 & 0 & 0 & 1,1 & 0 & 0 & 0 \\
\hline Wasmannia auropunctata (Roger, 1863) & 5,3 & 2,7 & 9,5 & 0,6 & 1,1 & 0 & 0 \\
\hline Wasmannia sp. 1 & 0 & 0 & 0 & 4,4 & 0 & 0 & 0 \\
\hline Wasmannia sp. 2 & 0 & 2,7 & 0 & 13,9 & 0 & 0 & 0 \\
\hline \multicolumn{8}{|l|}{ PONERINAE } \\
\hline Hypoponera sp. 1 & 0 & 0 & 4,8 & 1,1 & 0 & 0 & 0 \\
\hline Hypoponera sp. 2 & 1,3 & 0 & 4,8 & 0 & 0 & 0 & 0 \\
\hline Hypoponera sp. 3 & 0 & 0 & 4,8 & 0 & 0 & 0 & 0 \\
\hline Odontomachus chelifer (Latreille, 1802) & 12,0 & 0 & 4,8 & 8,3 & 0 & 0 & 0 \\
\hline Odontomachus meinerti Forel, 1905 & 1,3 & 0 & 0 & 7,8 & 0 & 0 & 0 \\
\hline Pachycondyla carinulata (Roger, 1861) & 0 & 0 & 0 & 0 & 0 & 4,8 & 2,7 \\
\hline Pachycondyla harpax (Fabricius, 1804) & 0 & 0 & 0 & 0,6 & 0 & 0 & 0 \\
\hline Pachycondyla magnifica Borgmeier, 1929 & 0 & 0 & 0 & 1,7 & 0 & 0 & 0 \\
\hline Pachycondyla aff. arhuaca & 0 & 0 & 0 & 0,6 & 0 & 0 & 0 \\
\hline Pachycondyla aff. cognata & 0 & 0 & 4,8 & 0 & 0 & 0 & 0 \\
\hline Pachycondyla striata & 12,0 & 0 & 4,8 & 27,2 & 0 & 0 & 0 \\
\hline Pachycondyla villosa (Fabricius, 1804) & 0 & 0 & 0 & 0 & 0 & 4,8 & 0 \\
\hline
\end{tabular}


Tabela I. Continuação

\begin{tabular}{|c|c|c|c|c|c|c|c|}
\hline \multicolumn{8}{|l|}{ FORMICINAE } \\
\hline Brachymyrmex sp. 1 & 0 & 18,9 & 0 & 1,7 & 7,8 & 9,5 & 0 \\
\hline Brachymyrmex sp. 2 & 1,3 & 2,7 & 4,8 & 1,7 & 0 & 0 & 0 \\
\hline Brachymyrmex sp. 3 & 4,0 & 10,8 & 9,5 & 6,7 & 1,1 & 0 & 10,8 \\
\hline Brachymyrmex sp. 4 & 0 & 5,4 & 0 & 0 & 2,2 & 0 & 0 \\
\hline Camponotus sericeiventris (Guzrin, 1838) & 2,7 & 0 & 0 & 0,6 & 0 & 9,5 & 10,8 \\
\hline Camponotus sp. 1 & 6,7 & 0 & 0 & 2,2 & 0 & 0 & 0 \\
\hline Camponotus sp. 2 & 0 & 0 & 4,8 & 1,1 & 0 & 0 & 0 \\
\hline Camponotus sp. 3 & 0 & 0 & 0 & 0,6 & 0 & 0 & 0 \\
\hline Camponotus sp. 4 & 0 & 0 & 0 & 0 & 0 & 4,8 & 0 \\
\hline Camponotus sp. 5 & 1,3 & 0 & 0 & 0 & 0 & 4,8 & 0 \\
\hline Camponotus sp. 6 & 0 & 0 & 0 & 0 & 1,1 & 4,8 & 2,7 \\
\hline Camponotus sp. 7 & 1,3 & 0 & 0 & 0 & 0 & 0 & 13,5 \\
\hline Camponotus sp. 9 & 0 & 5,4 & 0 & 0 & 0 & 0 & 0 \\
\hline Paratrechina sp. & 0 & 2,7 & 0 & 0 & 0 & 0 & 0 \\
\hline \multicolumn{8}{|l|}{ ECTATOMMINAE } \\
\hline Gnamptogenys annulata Mayr, 1887 & 0 & 0 & 0 & 0 & 0 & 0 & 2,7 \\
\hline Gnamptogenys aff. porcata & 0 & 0 & 0 & 3,9 & 0 & 0 & 0 \\
\hline Ectatoma edentatum Roger, 1863 & 2,7 & 0 & 0 & 11,7 & 0 & 0 & 0 \\
\hline \multicolumn{8}{|l|}{ HETEROPONERINAE } \\
\hline Heteroponera sp. & 0 & 0 & 0 & 0,6 & 0 & 0 & 0 \\
\hline \multicolumn{8}{|l|}{ DOLICHODERINAE } \\
\hline Linepithema iniquum Mayr, 1870 & 12,0 & 2,7 & 33,3 & 8,3 & 10,0 & 9,5 & 21,6 \\
\hline Tapinoma melanocephalum (Fabricius, 1793) & 0 & 0 & 0 & 0,6 & 2,2 & 0 & 2,7 \\
\hline \multicolumn{8}{|l|}{ ECITONINAE } \\
\hline Eciton sp. & 1,3 & 0 & 0 & 2,8 & 0 & 9,5 & 0 \\
\hline Nomamyrmex sp. 1 & 0 & 0 & 0 & 0,6 & 0 & 0 & 0 \\
\hline Nomamyrmex sp. 2 & 0 & 0 & 0 & 0,6 & 0 & 0 & 0 \\
\hline \multicolumn{8}{|l|}{ PSEUDOMYRMECINAE } \\
\hline Pseudomyrmex sp. 1 & 0 & 0 & 0 & 0 & 0 & 4,8 & 0 \\
\hline Pseudomyrmex sp. 2 & 0 & 0 & 0 & 0 & 0 & 19,1 & 0 \\
\hline Pseudomyrmex sp. 3 & 0 & 0 & 0 & 0 & 0 & 4,8 & 0 \\
\hline
\end{tabular}

Pheidole foi o gênero com maior riqueza de espécies (13), seguido por Solenopsis com nove e Camponotus com oito espécies. Do total de espécies registradas, 15 apresentaram apenas um indivíduo. Algumas espécies destacaram-se pela abundância e por aparecerem em todos os microhabitats amostrados. Linepithema iniquum (Mayr, 1870) e Pheidole sp. 3 foram coletadas em armadilhas no solo e na copa; nidificando em galhos finos e grossos; atraídas por iscas postas na serapilheira; coletadas sobre plantas do sub-bosque e no tronco das árvores (Tab. I). No geral, as amostras na serapilheira contribuíram com maior riqueza de espécies do que aquelas sobre plantas, mas essas últimas proporcionaram informações importantes, pois destacaram algumas espécies exclusivas neste ambiente (Tab. II).

Um total de 70 espécies de formigas foi registrado na serapilheira, sendo Pheidole sp. 3 a mais freqüente (40,32\% das amostras). Das oito subfamílias, apenas Pseudomyrmicinae não foi encontrada na serapilheira. Das técnicas utilizadas, o uso de armadilhas foi a mais representativa, com presença de formigas em $100 \%$ das amostras. A presença de formigas também foi elevada nas amostras de iscas sobre o solo (94\% do total). Todavia, todas as espécies coletadas nas iscas foram também coletadas em outras amostragens.

Nas amostras de galhos, foram coletadas 3.207 formigas em 1.337 galhos finos e 63 galhos grossos inspecionados. Nessas amostras foram obtidas 29 espécies, sendo que 23 efetivamente nidificavam nos galhos. Para as amostras de galhos finos, 3,3\% dos galhos apresentaram formigas em seu interior (1,4 galho com ninho $/ \mathrm{m}^{2}$ ). Para as amostras de galhos grossos, observaram-se formigas em 44,4\% desses ( 0,2 galho com ninho $\left./ \mathrm{m}^{2}\right)$. Brachymyrmex sp. 3 foi a espécie mais abundante e Brachymyrmex sp. 1 a mais frequiente nos galhos finos. Nos galhos grossos, Pheidole sp. 11 foi a mais abundante, Linephitema iniquum a mais freqüente. Das espécies registradas nos galhos, apenas para Hypoponera spp., Pachycondyla aff. cognata, Odontomachus chelifer e Solenopsis sp. 4 não foram encontradas colônias.

No total de amostras sobre plantas, obtiveramse 32 espécies, acrescentando 12 espécies não coletadas na serapilheira. No sub-bosque foram vistoriadas 206 plantas, das quais 42 apresentaram formigas. Coletaram-se formigas em apenas 21 armadilhas (18\% do total) utilizando os pitfalls içados até a copa das árvores. Já nos troncos das árvores vivas inspecionados, obtiveram-se formigas em 37 deles $(31 \%$ do total). 
Tabela II. Número de gêneros e espécies, total (T) e exclusivo (E), coletado nas diferentes amostras em floresta ombrófila de encosta na ilha da Marambaia, RJ (*, apenas as espécies identificadas).

\begin{tabular}{|c|c|c|c|c|c|}
\hline \multirow[t]{2}{*}{ Amostras } & \multicolumn{2}{|c|}{ Gêneros } & \multicolumn{2}{|c|}{ Espécies } & \multirow[t]{2}{*}{ Espécies exclusivas } \\
\hline & $\mathrm{T}$ & $\mathrm{E}$ & $\mathrm{T}$ & $\mathrm{E}$ & \\
\hline Serapilheira & 27 & 13 & 70 & 51 & \\
\hline Isca & 12 & 0 & 30 & 0 & - \\
\hline Galhos finos & 08 & 01 & 19 & 02 & Camponotus sp. 9, Paratrechina sp. \\
\hline Galhos grossos & 10 & 0 & 20 & 02 & Hypoponera sp. 3, Pachycondyla aff. cognata \\
\hline Pitfall & 26 & 09 & 61 & 24 & $\begin{array}{l}\text { *Cephalotes pallens, Mycocepurus smithi, Pachycondyla } \\
\text { harpax, P. magnifica, P. aff. arhuaca, Gnamptogenys aff. } \\
\text { porcata }\end{array}$ \\
\hline Plantas & 14 & 02 & 32 & 12 & \\
\hline Sub-bosque & 08 & 0 & 14 & 01 & Crematogaster nigropilosa \\
\hline Tronco & 12 & 0 & 17 & 02 & Acromyrmex aspersus, Gnamptogenys annulata \\
\hline Copa & 09 & 01 & 16 & 05 & $\begin{array}{l}\text { Pachycondyla villosa, Camponotus sp. 4, Pseudomyrmex sp. 1, } \\
\text { Pseudomyrmex sp. 2, Pseudomyrmex sp. } 3\end{array}$ \\
\hline
\end{tabular}

\section{DISCUSSÃO}

Em trabalho anterior, Meneguete et al. (1999) encontraram 21 gêneros e 39 morfoespécies de formigas em um local na ilha da Marambaia utilizando armadilhas de solo tipo pitfall. Apesar de suas coletas terem sido feitas ao longo de um ano, os autores utilizaram apenas quatro armadilhas. Este trabalho adiciona vários gêneros e dobra o número de espécies observadas anteriormente por ter utilizado um número maior de amostragens e técnicas diferentes daquela usada por MeneGuete et al. (1999). Se compararmos com estudos realizados em outras áreas de Mata Atlântica (Delabie et al., 2000a) e considerarmos que esta amostragem cobriu uma área de apenas 0,6 ha, a riqueza em espécies de formigas encontrada pode ser considerada alta.

$\mathrm{O}$ grande número de gêneros de formigas presente na ilha da Marambaia também chama atenção, já que WARD (2000) encontrou entre dois e 27 gêneros em 49 localidades na Região Neotropical, sendo que no geral as faunas continentais foram mais ricas em gêneros do que as faunas insulares. Cabe destacar que a ilha da Marambaia pode ser considerada um local com baixo isolamento. Ao longo da história de ocupação humana do local, desde o século XVII até o presente, várias introduções de espécies continentais podem ter ocorrido, principalmente na vertente Oeste, voltada para a baía de Sepetiba. Além disso, a ilha está conectada ao continente através de uma faixa estreita de areia, com cerca de $40 \mathrm{~km}$ de extensão, cuja origem sedimentar remonta ao Quaternário (PereIRA et al., 1990). Na ilha da Marambaia, houve, entretanto, um predomínio maior de espécies de Pheidole e Solenopsis. Enquanto esses dois gêneros respondem, mundialmente, por $22 \%$ das espécies, aqui eles corresponderam a $29 \%$ do total de espécies e $65 \%$ do total de indivíduos, mostrando forte dominância. Com efeito estes gêneros são geralmente ricos em espécies na fauna neotropical de formigas, mas seu predomínio maior, neste caso, deve ser por se tratar de uma fauna insular, ainda que com baixo grau de isolamento.

E possível que as temperaturas menores do inverno na ilha da Marambaia tenham sido responsáveis pela menor abundância e riqueza de espécies de formigas da serapilheira nas amostras de julho, aliadas, possivelmente, à menor ocorrência de chuvas nesta época do ano. Diferenças de temperatura similares às observadas na ilha entre março e julho (Pereira et al., 1990) podem causar uma variação de até $20 \%$ na atividade de algumas espécies de formigas (BESTELMEYER, 2000). Em geral, as condições de temperatura e umidade criam um envelope de restrições para as diferentes espécies de formigas, influenciando as suas atividades (HÖLLDOBLER \& WILSON, 1990), o que pode estar refletido nas coletas tomadas em diferentes épocas do ano.

O uso de técnicas e métodos variados acrescentou espécies àquelas coletadas exclusivamente com o pitfall de solo, que é a técnica mais amplamente utilizada (MAJER, 1997). A coleta de formigas que nidificam em galhos acrescentou informações sobre a biologia das espécies. Esta técnica contribuiu também para um maior conhecimento da riqueza local, uma vez que apresentou espécies exclusivas e possibilitou a identificação das espécies que se utilizam deste sítio para nidificação. A densidade de galhos finos colonizados por formigas na ilha da Marambaia foi bem superior ao encontrado por Carvalho \& Vasconcelos (2002) em floresta Amazônica $\left(0,22\right.$ galhos colonizados $\left./ \mathrm{m}^{2}\right)$, mas inferior ao verificado por B YRNe (1994) em uma floresta da Costa Rica (7,48 galhos colonizados $/ \mathrm{m}^{2}$ ). BYRNE (1994) sugere várias hipóteses para explicar as baixas frequiências de galhos colonizados por formigas. Entretanto, observar como variam essas frequiências ao longo do ano pode ser mais interessante, já que a oferta deste recurso também varia sazonalmente (MoRELLATO, 1992), e também a influência da qualidade da serapilheira, associada a diferentes estágios sucessionais de uma floresta, sobre a freqüência e diversidade de formigas que nidificam em galhos.

A proposta de utilizar armadilha tipo pitfall para coleta da fauna arborícola não encontrou suporte pelos resultados obtidos neste trabalho. Apenas um pequeno número de armadilhas conseguiu capturar formigas. Quando comparados com outros estudos de formicifauna de dossel (Schonberg et al., 2004; Schulz \& Wagner, 2002), o número de espécies registrado na copa das 
árvores na ilha da Marambaia foi pequeno. Isto ocorreu porque a instalação das armadilhas não ocorreu adequadamente devido às lianas, epífitas e do próprio sub-bosque. Esses problemas não ocorrem em outros tipos de vegetação com dossel mais baixo, como o cerrado, onde RiBAs et al. (2003) conseguiram amostrar com sucesso a fauna arborícola usando armadilhas do tipo pitfall. Na Mata Atlântica, conseguiu-se representar apenas uma pequena parcela das formigas das copas das árvores. Espécies tipicamente arborícolas, como as do gênero Pseudomymex e Pachycondyla villosa (LongIno, 2004), apareceram exclusivamente nestas amostras. Outras espécies arborícolas, como Gnamptogenys annulata e Pachycondyla carinulata (Roger, 1861) (LongInO, 2004), foram também registradas nos troncos das árvores. A amostragem da fauna arborícola na Mata Atlântica parece ser um problema ainda pendente. A técnica de nebulização com inseticidas piretróides tem recebido críticas, pois o sub-bosque pode agir como ponto de interceptação dos insetos atingidos pelo produto e, a altura elevada do dossel, possibilita os insetos mortos podem ser carregados pelo vento (ADIs et al., 1984). Apesar das limitações das técnicas para coleta de formigas sobre plantas, foi possível acrescentar um bom número de espécies àquele coletado na serapilheira.

A elevada riqueza de gêneros e espécies de formigas encontrada na ilha da Marambaia denota a importância do local como mantenedor de biodiversidade em ecossistemas no domínio da Mata Atlântica. Esse estudo incorpora informações sobre a diversidade de um grupo importante funcionalmente em ambientes tropicais e, juntamente com os projetos em andamento sobre outros componentes da fauna e da flora do local (Projeto Marambaia, 2005), contribui para o conhecimento e conservação da biodiversidade de um dos trechos mais ameaçados da Mata Atlântica no Estado do Rio de Janeiro.

Agradecimentos. Ao Programa de Apoio à Pesquisa em Espaços Delimitados (PAPED-UFRRJ), pela bolsa de iniciação científica concedida ao primeiro autor. Ao Centro de Adestramento da Ilha da Marambaia (CADIM, Marinha do Brasil) pelo transporte, alojamento e alimentação proporcionados à equipe de pesquisa. A todos os colegas que auxiliaram nos trabalhos de campo e laboratório e a Alex Wild (EUA), que identificou Linepithema iniquum em nossas amostras. Ao Prof. Roberto de Xerez, representante da UFRRJ no convênio com o CADIM, pelo apoio à realização deste trabalho. Aos Profs. Roberto de Xerez, Lenício Gonçalves (UFRRJ) e dois revisores anônimos que fizeram importantes sugestões em versões anteriores do manuscrito. Ao CNPq pela bolsa de pesquisa a AJMN.

\section{REFERÊNCIAS BIBLIOGRÁFICAS}

Adis, J.; Lubin, Y. \& Montgomery, G. 1984. Arthropods from the canopy of inundated and terra firme forest near Manaus, with critical considerations on the pyrethrum-fogging technique. Studies on Neotropical Fauna and Environment 19(4):223-236.

Agosti, D. \& Alonso, L. E. 2000. The ALL protocol: selected case studies. In: Agosti, D.; Majer, J. D.; Alonso, L. E. \& Schultz, T. R. eds. Ants: standard methods for measuring and monitoring biodiversity. Washington, Smithsonian Institution. p.204-206.

Bestelmeyer, B. T. 2000. The trade-off between thermal tolerance and behavioural dominance in a subtropical South American ant community. Journal of Animal Ecology 69(6):998-1009.
Byrne, M. M. 1994. Ecology of twig-dwelling ant in wet lowland tropical Forest. Biotropica 26(1):61-72.

Carvalho, K. S. \& Vasconcelos, H. L. 2002. Comunidade de formigas que nidificam em pequenos galhos da serapilheira em floresta da Amazônia Central, Brasil. Revista Brasileira de Entomologia 46(2): 115-121.

Davidson, D. W.; Cook, S. C.; Snelling, R. R. \& Chua, T. H. 2003. Explaining the abundance of ants in lowland tropical rainforest canopies. Science 300(5621):969-972.

Delabie, J. H. C.; Agosti, D. \& Nascimento, I. C. 2000a. Litter ant communities of the Brazilian Atlantic rain forest region. In: Agosti, D.; Majer, J. D.; Alonso, L. E. \& Schultz, T. R. eds. Sampling ground-dwelling ants: case studies from the Worlds' rain forests. Perth, Curtin University School of Environmental Biology. p.1-17. (Bulletin, 18)

Delabie, J. H. C.; Fisher, B. L.; Majer, J. D. \& Wright, I. W 2000b. Sampling effort and choice of methods. In: Agosti, D. Majer, J. D.; Alonso, L. E. \& Schultz, T. R. eds. Ants: standard methods for measuring and monitoring biodiversity. Washington, Smithsonian Institution. p.145-154

Greenslade, P. J. M. 1973. Sampling ants with pitfall traps: digging-in effects. Insect Sociaux 20(4):345-353.

Hölldobler, B. \& Wilson, E. O. 1990. The ants. Cambridge, Harvard University. 732p.

Lewinsohn, T. M.; Freitas, A. V. L. \& Prado, P. I. 2005. Conservation of terrestrial invertebrates and their habitats in Brazil. Conservation Biology 19(3):640-645.

Longino, J. T. 2004. Ants of Costa Rica. Disponível em: <http:/ /www.evergreen.edu/ants/antsofcostarica.html>. Acesso em: 01.07.2005.

Longino, J. T. \& Colwell, R. K. 1997. Biodiversity assessment using structured inventory: capturing the ant fauna of a tropical rain forest. Ecological Applications 7(4):12631277.

Magurran, A. E. 1988. Ecological diversity and its measurement. London, Croom Helm. 178p.

MAJER, J. D. 1997. The use of pitfall traps for sampling ants - a critique. Memoirs of the Museum of Victory 56(2):323-329.

Meneguete, P. S.; Xerez, R. \& Mayhé-Nunes, A. J. 1999. Atratividade de quatro tipos de iscas utilizadas para a captura de formigas (Hymenoptera: Formicidae) na Praia da Armação, Ilha da Marambaia, Mangaratiba, Rio de Janeiro. Acta Biologica Leopoldensia 21(2):221-228.

MMA/SBF. 2003. Áreas prioritárias para a conservação, utilização sustentável e repartições de benefícios da biodiversidade brasileira. Projeto de conservação e utilização sustentável da diversidade biológica brasileira PROBIO. Brasília, Ministério do Meio Ambiente/SBF. 340p.

Morellato, L. P. C. 1992. Sazonalidade e dinâmica de ecossistemas florestais da Serra do Japi. In: Morellato, L. P. C. \& LeitãoFilho, H. F. eds. História natural da Serra do Japi: ecologia e preservação de uma área florestal no sudeste do Brasil. Campinas, UNICAMP. p.97-110.

Myers, N.; Mittermeier, R. E.; Mittermeier, C. G.; Fonseca, G. A. B. \& Kent, J. 2000. Biodiversity hostpot for conservation priorities. Nature 403(6772):853-858.

Pereira, L. A.; Xerez, R. \& Pereira, A. M. C. 1990. Ilha da Marambaia (Baía de Sepetiba, RJ): Resumo fisiográfico, histórico e importância ecológica atual. Ciência e Cultura 42(5/6):384-389.

Projeto Marambaia. 2005. Estudos integrados em florestas de restinga no litoral sul-fluminense. Departamento de Botânica, Instituto de Biologia, Universidade Federal Rural do Rio de Janeiro. Disponível em:<http://projetomarambaia.cjb.net/>. Acesso em:01.07.2005

Ribas, C. R.; Schoereder, J. H.; Pic, M. \& Soares, S. M. 2003. Tree heterogeneity, resource availability, and larger scale processes regulating arboreal ant species richness. Austral Ecology 28(3):305-314.

Rocha, C. F. D.; Bergallo, H. G.; Alves, M. A. S. \& Van-Sluys, M. 2003. A biodiversidade nos grandes remanescentes florestais do Estado do Rio de Janeiro e nas restingas da Mata Atlântica. São Carlos, RiMa. 134p.

SchonberG, L. A.; Longino, J. T.; NADKARni, N. M. \& Yanoviak, S. P. 2004. Arboreal ant species richness in primary forest secundary forest, and pasture habitats of a tropical montane 
landscape. Biotropica 36(3):402-409.

Schultz, T. R. \& McGlynn, T. P. 2000. The interactions of ants with other organisms. In: Agosti, D.; Majer, J. D.; Alonso, L. E. \& Schultz, T. R. eds. Ants: standard methods for measuring and monitoring biodiversity. Washington, Smithsonian Institution. p.35-44.

Schulz, A. \& Wagner, T. 2002. Influence of forest type and tree species on canopy ants (Hymenoptera: Formicidae) in Budongo
Forest, Uganda. Oecologia 133(2):224-232.

WARD, P. S. 2000. Broad-scale patterns of diversity in leaf litter ant communities. In: Agosti, D.; Majer, J. D.; Alonso, L. E. \& Schultz, T. R. eds. Ants: standard methods for measuring and monitoring biodiversity. Washington, Smithsonian Institution. p.99-121

ZaR, J. H. 1999. Biostatistical Analysis. New Jersey, Prentice Hall. 663p.

Recebido em julho de 2005. Aceito em outubro de 2006. ISSN 0073-4721

Artigo disponível em: www.scielo.br/isz

Iheringia, Sér. Zool., Porto Alegre, 97(1):103-110, 30 de março de 2007 